\title{
Tropical linear spaces and tropical convexity
}

\author{
Simon Hampe* \\ Institut fr Mathematik \\ Technische Universitt Berlin \\ Berlin, Germany \\ hampe@math.tu-berlin.de
}

Submitted: May 21, 2015; Accepted: Dec 9, 2015; Published: Dec 22, 2015

Mathematics Subject Classifications: 14T05, 52A99

\begin{abstract}
In classical geometry, a linear space is a space that is closed under linear combinations. In tropical geometry, it has long been a consensus that tropical varieties defined by valuated matroids are the tropical analogue of linear spaces. It is not difficult to see that each such space is tropically convex, i.e. closed under tropical linear combinations. However, we will also show that the converse is true: Each tropical variety that is also tropically convex is supported on the complex of a valuated matroid. We also prove a tropical local-to-global principle: Any closed, connected, locally tropically convex set is tropically convex.
\end{abstract}

\section{Introduction}

It has long been a consensus what the tropical analogue of a linear space should be. Sturmfels showed in [25] that the tropicalization of a complex variety defined by linear equations depends only on a matroid $M$ associated to these equations. One can give this tropical variety, the matroidal fan or Bergman fan of $M$ in various purely combinatorial ways: E.g. through its circuits, its bases or its lattice of flats [9, 4]. One can do this for any matroid, though only realizable matroids yield tropical varieties that are tropicalizations of algebraic linear spaces. In the case of fields with a nontrivial valuation, the tropicalization of a linear space is defined by a valuated matroid $(M, w)$. This notion was originally introduced by Dress and Wenzel [8]. It is given by a matroid $M$ and the additional data of a valuation $w$ on its bases. Again, the tropical space can be defined for any such object and it was soon established that the associated tropical varieties should be called tropical linear spaces.

*Partially supported by DFG grants 4797/1-2 and JO366/3-2 (This grant is part of the DFG priority project SPP 1489, see http://www. computeralgebra.de) and EPSRC grant EP/I008071/1. 
This terminology is further justified by the fact that being a tropical linear space is equivalent to this space having degree one: This means that it intersects the linear space of complementary dimension associated to the uniform matroid in exactly one point (where intersection is to be understood as stable intersection). A proof of this can be found in [10], though the statement seems to have been known for longer (see for example [14]).

Someone familiar with tropical arithmetic might expect a different definition. In the algebraic world, a linear space is simply a space that is closed under linear combinations. On the tropical side, addition and multiplication are replaced by $\oplus=\max$ and $\odot=+$. Using these one can define tropical vector addition and tropical scalar multiplication. One might then be tempted to define a tropical linear space as a space that is closed under tropical linear combinations. This property is well-known under the name of tropical convexity. At first glance, this might seem to be a misnomer, but its justification quickly becomes clear when looking at the corresponding literature. It turns out that classical and tropical convexity are closely related. Develin and Sturmfels first introduced the concept into the tropical world [6] and proved - among other things - that there is a tropical Farkas' Lemma. Gaubert and Katz prove in [13] that tropical polytopes, i.e. the convex hulls of finitely many points, can also be written as the intersection of finitely many tropical halfspaces. Develin and $\mathrm{Yu}$ showed that tropical polytopes are tropicalizations of actual polytopes [7]. Tropical convexity has connections to many fields, such as graph theory, optimization, resolutions of monomial ideals or subdivisions of polytopes (see for example $[1,2,5,11,16])$.

It becomes readily apparent that simply demanding tropical convexity will not in general produce sets that are tropical linear spaces in the approved sense. However, when adding the prerequisite that the set be supported on a tropical variety, i.e. be a balanced polyhedral complex, the statement becomes true. In fact, it was already well-known that any tropical linear space (meaning a space associated to a valuated matroid) is a tropical variety supported on a tropically convex set. In this paper, we prove that the converse is also true:

Theorem 1.1. Let $X$ be a tropical variety in $\mathbb{R}^{n} / 1$. Then $|X|$ is tropically convex, if and only if $|X|=B(M, w)$ for some valuated matroid $(M, w)$ on $[n]$. In other words, $X$ is the quotient of a space closed under tropical linear combinations if and only if $X$ is supported on a tropical linear space.

In Section 2 we will review basic definitions and facts about tropical convexity, tropical varieties, valuated matroids and their associated varieties. We also include a proof of the fact that a tropical linear space is tropically convex. In Section 3 we collect results about general tropically convex complexes (i.e. that do not require balancing). We show that tropical convexity passes to recession fans and is locally preserved. We also prove that any tropically convex fan of dimension $d$ is contained in the $d$-skeleton of the normal fan of the permutohedron. Section 4 then contains the actual proof of Theorem 1.1. We prove the statement first for fans and trivially valuated matroids. The general result then follows (with a bit more work) from the fact that being a tropical linear space is also equivalent to having a recession fan that is a tropical linear space. In section 5 , we prove a tropical 
local-to-global convexity theorem:

Theorem 1.2. Let $X \subseteq \mathbb{R}^{n} / 1$ be a closed, connected set. If $X$ is locally tropically convex, then $X$ is tropically convex.

From this we deduce that being a tropical linear space is a local property.

\section{Preliminaries}

Convention. Throughout this paper we use $\oplus=\max$ as tropical addition. Of course, all results still hold in the min-world, one simply has to "invert" the definition of tropical linear spaces as well. We also write $\odot=+$ for tropical multiplication.

\subsection{Tropical convexity}

Definition 2.1. Let $x, y \in \mathbb{R}^{n}$. We define the tropical sum of $x$ and $y$ to be the componentwise tropical sum: $x \oplus y:=\left(x_{1} \oplus y_{1}, \ldots, x_{n} \oplus y_{n}\right)$. Similarly, for $\lambda \in \mathbb{R}, x \in \mathbb{R}^{n}$ we define tropical scalar multiplication to be componentwise tropical multiplication: $\lambda \odot x:=$ $\left(x_{1}+\lambda, \ldots, x_{n}+\lambda\right)$.

A subset $S$ of $\mathbb{R}^{n}$ is called tropically convex, if for all $x, y \in S, \lambda, \mu \in \mathbb{R}$ we have

$$
(\lambda \odot x) \oplus(\mu \odot y) \in S .
$$

The tropical convex hull of a set $T$, denoted by $\operatorname{tconv}(T)$, is the smallest tropically convex set containing $T$. By [6] it is equal to the set of all tropical linear combinations of elements in $T$.

Remark 2.2. It is easy to see that any tropically convex set is invariant under translation by multiples of $(1, \ldots, 1)$. Hence it is customary to consider subsets $S^{\prime}$ of the tropical projective torus $\mathbb{R}^{n} / \mathbf{1}$, where $\mathbf{1}:=\langle(1, \ldots, 1)\rangle$. We say that such a set $S^{\prime}$ is tropically convex, if its preimage under the quotient map $\mathbb{R}^{n} \rightarrow \mathbb{R}^{n} / \mathbf{1}$ is. Note, however, that tropical arithmetic operations are not actually well-defined on $\mathbb{R}^{n} / \mathbf{1}$.

Lemma 2.3 ([6, Proposition 3]). Let $x, y \in \mathbb{R}^{n} / \mathbf{1}$. Then the tropical convex hull of $\{x, y\}$ is of the form $\left(\bigcup_{i=1}^{k} l_{i}\right)$, where the $l_{i}$ are consecutive line segments connecting $x$ and $y$, whose slopes are linearly independent $(0,1)$-vectors. Furthermore, the number of these line segments is $k:=\left|\left\{x_{i}-y_{i} ; i=1, \ldots, n\right\}\right|-1$.

Remark 2.4. Let us make this statement more concrete: Every element $x \in \mathbb{R}^{n} / \mathbf{1}$ has a well-defined heterogeneity:

$$
\operatorname{het}(x):=\left|\left\{x_{i} ; i=1, \ldots, n\right\}\right| .
$$

We can also define a partition associated to $x, \operatorname{part}(x)=\left(I_{1}, \ldots, I_{\text {het }(x)}\right)$ of $[n]$ ordering the entries of $x$ descendingly. More precisely:

- For any $j=1, \ldots, \operatorname{het}(x)$ and $k, l \in I_{j}$, we have $x_{k}=x_{l}=: x\left(I_{j}\right)$. 
- If $j<j^{\prime}$, then $x\left(I_{j}\right)>x\left(I_{j^{\prime}}\right)$.

Now fix representatives $x, y$ of two elements in $\mathbb{R}^{n} / \mathbf{1}$ and set $\Delta:=\Delta(x, y)=y-x$. Assume that $\operatorname{part}(\Delta)=\left(I_{1}, \ldots, I_{s}\right)$. For any set $F \subseteq[n]$ we write

$$
e_{F}:=\sum_{i \in F} e_{i}
$$

If we define $F_{j}:=\bigcup_{i=1}^{j} I_{i}$ for $j=1, \ldots, s-1$, then the tropical convex hull tconv $\{x, y\}$ consists of the line segments connecting points $x=p_{1}, \ldots, p_{s}$, where for $j>1$ we set

$$
p_{j}:=p_{j-1}+\left(\Delta\left(I_{j-1}\right)-\Delta\left(I_{j}\right)\right) e_{F_{j-1}}=x+\sum_{i=2}^{j}\left(\Delta\left(I_{i-1}\right)-\Delta\left(I_{i}\right)\right) e_{F_{i-1}} .
$$

In particular $p_{s}=\Delta\left(I_{s}\right) \odot y \equiv y$ in $\mathbb{R}^{n} / \mathbf{1}$ and the slope of the line segment $\left[p_{j}, p_{j+1}\right]$ is $e_{F_{j}}$.

Example 2.5. Let $n=3$. We choose a representative of each element in $\mathbb{R}^{3} / \mathbf{1}$ by setting the first coordinate to be 0 . Choose $x=(0,-1,-1), y=(0,2,1)$. Then $\Delta=(0,3,2)$, so we have $\operatorname{part}(\Delta)=(\{2\},\{3\},\{1\})$. In particular, we get

$$
\begin{aligned}
& p_{1}=x=(0,-1,-1), \\
& p_{2}=x+(3-2) e_{\{2\}}=x+(0,1,0)=(0,0,-1), \\
& p_{3}=(0,0,-1)+(2-0) e_{\{2,3\}}=(0,0,-1)+2 \cdot(0,1,1)=(0,2,1)=y .
\end{aligned}
$$

Note that there is a nice geometric way to construct this: Draw a min-tropical line at each vertex. This induces a subdivision of the plane and the tropical convex hull then consists of the bounded cells of this subdivision. This also works for the tropical convex hull of an arbitrary (finite) number of vertices and in arbitrary dimensions, see [6, Theorem 15].

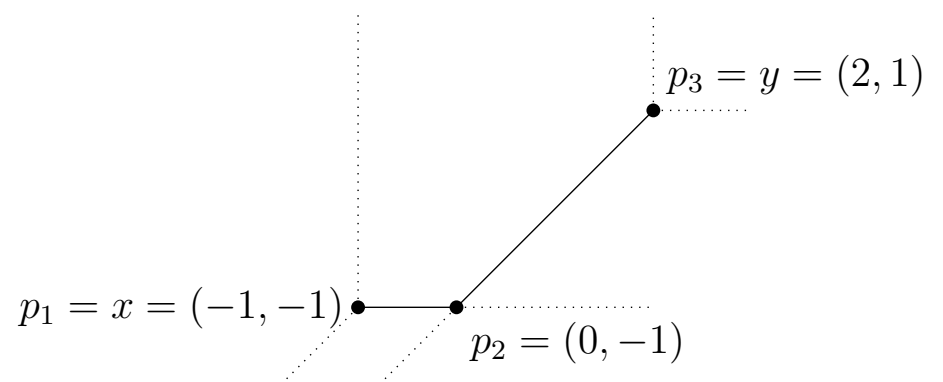

Figure 1: Constructing the tropical convex hull of two points. 


\subsection{Tropical varieties}

We will only give the very basic definitions necessary for our purpose. For a general introduction to tropical geometry, see for example $[14,18,17]$.

Definition 2.6. Let $X$ be a pure $d$-dimensional rational polyhedral complex in $\mathbb{R}^{n} / \mathbf{1}$. We will denote the support of $X$ by $|X|:=\bigcup_{\sigma \in X} \sigma$. For a cell $\rho$ of $X$ we define $V_{\rho}:=$ $\langle a-b ; a, b \in \rho\rangle$ to be the vector space associated to the affine space spanned by $\rho$ and we write $\Lambda_{\rho}:=V_{\rho} \cap \mathbb{Z}^{n} / \mathbf{1}$ for its lattice.

- Let $\sigma \in X^{(d)}:=\{\sigma \in X ; \operatorname{dim}(\sigma)=d\}$ and assume $\tau \subseteq \sigma$ is a face of dimension $d-1$. The primitive normal vector of $\tau$ with respect to $\sigma$ is defined as follows: By definition there is a linear form $g$ such that its minimal locus on $\sigma$ is $\tau$. Then there is a unique generator of $\Lambda_{\sigma} / \Lambda_{\tau} \cong \mathbb{Z}$, denoted by $u_{\sigma / \tau}$, such that $g\left(u_{\sigma / \tau}\right)>0$.

- A tropical variety $(X, \omega)$ is a pure, rational polyhedral complex $X$ together with a weight function $\omega: X^{\max } \rightarrow \mathbb{N}_{>0}$ (where $X^{\max }$ denotes the set of maximal polyhedral cells of $X$ ) fulfilling the balancing equation at each codimension one cell $\tau$ :

$$
\sum_{\sigma>\tau} \omega(\sigma) u_{\sigma / \tau}=0 \bmod V_{\tau}
$$

Note that we will consider two tropical varieties to be equivalent if they have a common refinement respecting the weight functions. A tropical fan is a tropical variety whose polyhedral structure can be represented by a fan.

- For a polyhedral cell $\sigma$, we denote by

$$
\begin{aligned}
\operatorname{rec}(\sigma) & :=\left\{v \in \mathbb{R}^{n} / \mathbf{1}: x+\mathbb{R}_{\geqslant 0} v \subseteq \sigma \text { for all } x \in \sigma\right\} \\
& =\left\{v \in \mathbb{R}^{n} / \mathbf{1}: \exists x \in \sigma \text { such that } x+\mathbb{R}_{\geqslant 0} v \subseteq \sigma\right\}
\end{aligned}
$$

its recession cone. One can choose a refinement of a polyhedral complex $X$ such that

$$
\operatorname{rec}(X):=\{\operatorname{rec}(\sigma) ; \sigma \in X\}
$$

is a fan and we will call that the recession fan of $X$. If $(X, \omega)$ is a tropical variety, so is $\left(\operatorname{rec}(X), \omega_{\text {rec }}\right)$, where

$$
\omega_{\operatorname{rec}}(\rho)=\sum_{\sigma: \operatorname{rec}(\sigma)=\rho} \omega(\sigma) .
$$

(see [22, p. 61] for a proof of this. It also follows implicitly from [3, Theorem 5.4].)

- A $d$-dimensional tropical variety $\left(X, \omega_{X}\right)$ is called irreducible, if every $d$-dimensional variety $\left(Y, \omega_{Y}\right)$ with $|Y| \subseteq|X|$ is an integer multiple of $X$, i.e. $|Y|=|X|$ and (assuming we have chosen a common refinement) $\omega_{Y}=k \cdot \omega_{X}$ for some $k \in \mathbb{N}$. We also write this as $Y=k \cdot X$. 
- Let $X$ be a tropical variety and $p \in|X|$. By refining, we can assume without loss of generality that $p$ is a vertex of $X$. We define the Star of $X$ at $p$ to be the fan

$$
\operatorname{Star}_{X}(p):=\left\{\mathbb{R}_{\geqslant 0} \cdot(\sigma-p) ; p \in \sigma\right\}
$$

with weight function $\omega_{\text {Star }}\left(\mathbb{R}_{\geqslant 0}(\sigma-p)\right)=\omega_{X}(\sigma)$. It is easy to see that this is a tropical fan and that its support is equal to

$$
\left\{v \in \mathbb{R}^{n} / \mathbf{1} \text {; There is } r_{v}>0 \text { such that } p+\alpha v \in|X| \text { for all } \alpha \in\left[0, r_{v}\right]\right\} .
$$
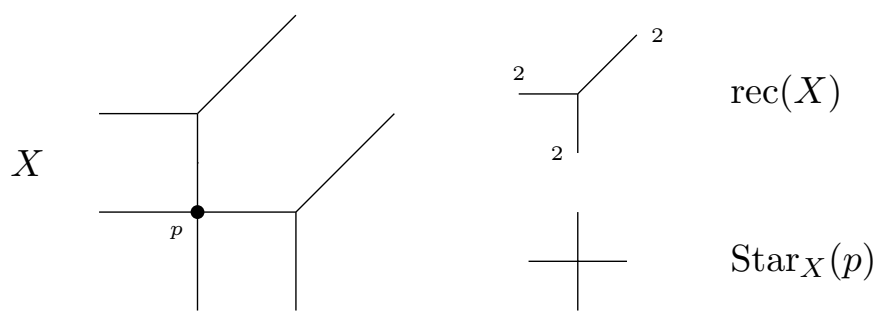

Figure 2: Forming the recession fan and the Star of $X$ at a point. Note that, while $\operatorname{rec}(X)$ is supported on a tropical linear space, it has nontrivial weights, so Theorem 2.11 does not apply.

\subsection{Tropical linear spaces}

We will assume that the reader is familiar with the basic notions of matroid theory (see [21] for a comprehensive study of the topic). For a study of tropical linear spaces see for example $[11,23,17,24]$. To quickly recap the matroid terminology we will mostly use: A circuit is a minimal dependent set and a flat is a closed set, i.e. adding any element increases the rank. Note that we will assume all matroids to be loopfree.

Dress and Wenzel [8] generalized the notion of a matroid to that of a valuated matroid:

Definition 2.7. A valuated matroid $(M, w)$ is a matroid $M$ on a set $E=\{1, \ldots, n\}$ together with a valuation $w: \mathcal{B} \rightarrow \mathbb{R}$ on its set of bases $\mathcal{B}$ fulfilling the tropical Plcker relations:

For all $B_{1}, B_{2} \in \mathcal{B}$ and every $u \in B_{1}$, there exists a $v \in B_{2}$, such that both $B_{1}-u+v$ and $B_{2}-v+u$ are bases and

$$
\left(w\left(B_{1}\right) \odot w\left(B_{2}\right)\right) \oplus\left(w\left(B_{1}-u+v\right) \odot w\left(B_{2}-v+u\right)\right)=w\left(B_{1}-u+v\right) \odot w\left(B_{2}-v+u\right) .
$$

Remark 2.8. As in classical matroid theory, there are various equivalent ways of defining a valuated matroid. Another way, discovered by Murota and Tamura [19] is via valuated circuits. A circuit valuation is obtained by choosing a vector $v_{C} \in(\mathbb{R} \cup(-\infty) / \mathbf{1})^{n}$ for each circuit $C$ of $M$ such that the following are fulfilled:

- $C=\left\{i \in[n] ;\left(v_{C}\right)_{i} \neq-\infty\right\}$ for all circuits $C$. 
- Let $C, C^{\prime}$ be circuits and assume $i \in C \cap C^{\prime}, j \in C \backslash C^{\prime}$. Choose representatives of $v_{C}, v_{C^{\prime}}$ such that $\left(v_{C}\right)_{i}=\left(v_{C^{\prime}}\right)_{i}$. Then there exists a circuit $D$ and a representative of $v_{D}$ such that $i \notin D,\left(v_{D}\right)_{j}=\left(v_{C}\right)_{j}$ and $v_{D} \oplus v_{C} \oplus v_{C^{\prime}}=v_{C} \oplus v_{C^{\prime}}$.

The paper [19] shows that both axiom sets are cryptomorphic. More precisely, given a valuation $w: \mathcal{B} \rightarrow \mathbb{R}$ on the bases, a circuit valuation can be defined in the following way: Let $C$ be a circuit. Then $C$ is the fundamental circuit with respect to some basis $B$ and an element $i \notin B$. We set

$$
\left(v_{C}^{w}\right)_{j}:=w(B-j+i)-w(B)
$$

where $w\left(B^{\prime}\right)=-\infty$, if $B^{\prime}$ is not a basis. We will consider any valuated matroid $(M, w)$ to be equipped with this circuit valuation.

Definition 2.9. One can define a polyhedral structure on the set

$$
B(M, w):=\left\{x \in \mathbb{R}^{n} / \mathbf{1} ; \max _{i \in C}\left\{x_{i}+\left(v_{C}^{w}\right)_{i}\right\} \text { is assumed at least twice } \forall \text { circuits } C\right\} .
$$

and assigning weight 1 to each maximal cell we obtain a tropical variety (a proof can be found in [17, Theorem 4.4.5]), which we also denote by $B(M, w)$. A tropical linear space is a tropical variety of this form.

Remark 2.10. It turns out that we essentially only need to consider trivial valuations (i.e. $w \equiv 0$ ) to prove our theorem, in which case we obtain a polyhedral fan, the matroidal fan or Bergman fan of a matroid $M$ :

$$
B(M):=\left\{x \in \mathbb{R}^{n} / \mathbf{1} ; \max _{i \in C} x_{i} \text { is assumed at least twice for all circuits } C \text { of } M\right\} .
$$

Note that for any valuation $w$ on a matroid $M, B(M)=\operatorname{rec}(B(M, w))$. The key fact that will allow us to reduce the problem to matroidal fans will be the following:

Theorem 2.11. Let $X$ be a tropical variety. Then the following are equivalent:

- $X=B(M, w)$ for a valuated matroid $(M, w)$.

- $\operatorname{rec}(X)=B(M)$.

This follows from the two facts that being a tropical linear space is equivalent to having degree one [10, Theorem 6.5] and that a tropical variety and its recession fan have the same degree (see for example the argument in the proof of [17, Theorem 4.4.5]).

It has been shown that $B(M)$ has several possible representations as a polyhedral fan. We will be working with the structure induced by the flats of $M$ - this is the finest fan structure of $B(M)$ that usually occurs in the literature: 
Definition 2.12. For a set $F \subseteq[n]$ we write $v_{F}:=-e_{F}=-\sum_{i \in F} e_{i}$.

Let $\mathcal{F}$ be the set of flats of $M$. For any chain $\mathcal{C}=\left(F_{1}, \ldots, F_{d}=E\right)$, where $\emptyset \subsetneq F_{1} \subsetneq$ $\cdots \subsetneq F_{d}=E$ and $F_{i} \in \mathcal{F}$ for all $i$, we define a polyhedral cone:

$$
\operatorname{cone}(\mathcal{C}):=\left\{\sum_{i=1}^{d-1} \lambda_{i} v_{F_{i}} ; \lambda_{1}, \ldots, \lambda_{d-1} \geqslant 0\right\}
$$

If we go through all chains of flats in $M$, the corresponding cones obviously form a fan and by [4] the support of this fan is $B(M)$.

Remark 2.13. One can also retrieve the matroid from its Bergman fan. It is a well-known fact that its set $\mathcal{F}$ of flats is $\left\{F \subseteq[n] ; v_{F} \in B(M)\right\}$. To see this, assume $v_{F} \in B(M)$. By [9] this is the same as saying that the set of bases $B$ of $M$ such that $|B \cap F|$ is maximal covers all of $E$, as these are the bases of minimal weight with respect to $v_{F}$. But that implies that $F$ is a flat: If $i \notin F$, there is a basis $B$ containing $i$ and having maximal intersection with $F$, so $\operatorname{rank}(F+i)=|B \cap(F+i)|=|B \cap F|+1=\operatorname{rank}(F)+1$.

The following has been known for long, but we include the proof here for completeness:

Proposition 2.14. Let $(M, w)$ be a valuated matroid of rank $r$ on $n$ elements. Then $B(M, w)$ is tropically convex.

Proof. Let $x, y \in B((M, w))$ and $\lambda, \mu \in \mathbb{R}$. Let

$$
z:=\lambda \odot x \oplus \mu \odot y=\left(\max \left\{x_{i}+\lambda, y_{i}+\mu\right\}\right)_{i=1, \ldots, n} .
$$

Let $C \subseteq[n]$ be a circuit of $M$. We will denote the corresponding valuation by $v_{C}$. In that case $\max _{i \in C}\left\{x_{i}+\left(v_{C}\right)_{i}\right\}, \max _{i \in C}\left\{y_{i}+\left(v_{C}\right)_{i}\right\}$ are both assumed twice, i.e. there exist $i_{1} \neq i_{2}, j_{1} \neq j_{2}$ such that

$$
\begin{aligned}
x_{C} & :=\max _{i \in C}\left\{x_{i}+\left(v_{C}\right)_{i}\right\}=x_{i_{1}}+\left(v_{C}\right)_{i_{1}}=x_{i_{2}}+\left(v_{C}\right)_{i_{2}} \\
y_{C} & :=\max _{i \in C}\left\{y_{i}+\left(v_{C}\right)_{i}\right\}=y_{j_{1}}+\left(v_{C}\right)_{j_{1}}=y_{j_{2}}+\left(v_{C}\right)_{j_{2}}
\end{aligned}
$$

We will assume without restriction that $y_{C}+\mu \geqslant x_{C}+\lambda$. Thus, $z_{j_{1}}+\left(v_{C}\right)_{j_{1}}=z_{j_{2}}+\left(v_{C}\right)_{j_{2}}=$ $y_{C}+\mu$. Let $k \in C$ be arbitrary. Then

$$
\begin{aligned}
z_{k}+\left(v_{C}\right)_{k} & =\max \left\{x_{k}+\left(v_{C}\right)_{k}+\lambda, y_{k}+\left(v_{C}\right)_{k}+\mu\right\} \\
& \leqslant \max \left\{x_{C}+\lambda, y_{C}+\mu\right\} \\
& =y_{C}+\mu=z_{j_{1}}+\left(v_{C}\right)_{j_{1}} .
\end{aligned}
$$

In particular, the maximum $\max _{i \in C}\left\{z_{i}+\left(v_{C}\right)_{i}\right\}$ is assumed twice (at $j_{1}$ and $j_{2}$ ). 


\section{Tropically convex complexes}

Proposition 3.1. Let $X$ be a polyhedral complex and assume $|X|$ is tropically convex. Then $|\operatorname{rec}(X)|$ is tropically convex as well.

Proof. Assume $v, v^{\prime} \in|\operatorname{rec}(X)|$. We can reformulate this as the fact that there exist $p, p^{\prime} \in|X|$ such that $p+\mathbb{R}_{\geqslant 0} v, p^{\prime}+\mathbb{R}_{\geqslant 0} v^{\prime} \subseteq|X|$. For $\alpha>0$ we write $q_{\alpha}:=p+\alpha v, q_{\alpha}^{\prime}:=$ $p^{\prime}+\alpha v^{\prime}$ and $\Delta_{\alpha}:=q_{\alpha}^{\prime}-q_{\alpha}, \nu:=v^{\prime}-v$. It is easy to see that we can choose $\alpha$ large enough such that part $\left(\Delta_{\alpha}\right)$ remains constant and is a refinement of part $(\nu)$, by which we mean that if $\nu_{i}<\nu_{j}$, this implies $\left(\Delta_{\alpha}\right)_{i}<\left(\Delta_{\alpha}\right)_{j}$. Assume we have fixed such an $\alpha$ and that part $\left(\Delta_{\alpha}\right)=\left(I_{1}, \ldots, I_{s}\right)$. Now as in Remark 2.4 we see that the tropical convex hull tconv $\left\{q_{\alpha}, q_{\alpha}^{\prime}\right\}$ consists of line segments connecting points

$$
p_{j}^{\alpha}:=q_{\alpha}+\sum_{i=2}^{j}\left(\Delta_{\alpha}\left(I_{i-1}\right)-\Delta_{\alpha}\left(I_{i}\right)\right) e_{F_{i-1}},
$$

where $F_{i}=\bigcup_{k \leqslant i} I_{k}$. Now let $\beta>\alpha$. We calculate that for all $j=1, \ldots, s$ we have

$$
\begin{aligned}
p_{j}^{\beta}-p_{j}^{\alpha} & =\left(q_{\beta}-q_{\alpha}\right)+\sum_{i=2}^{j}\left(\left(\Delta_{\beta}\left(I_{i-1}\right)-\Delta_{\beta}\left(I_{i}\right)\right)-\left(\Delta_{\alpha}\left(I_{i-1}\right)-\Delta_{\alpha}\left(I_{i}\right)\right)\right) e_{F_{i-1}} \\
& =(\beta-\alpha) \underbrace{\left(v+\sum_{i=2}^{j}\left(\nu\left(I_{i-1}\right)-\nu\left(I_{i}\right)\right) e_{F_{i-1}}\right)}_{=: r_{j}} .
\end{aligned}
$$

Note that $r_{1}, \ldots, r_{s}$ are exactly the vertices of the line segments forming $\operatorname{tconv}\left\{v, v^{\prime}\right\}$ (some of them may be the same, as part $\left(\Delta_{\alpha}\right)$ can be strictly finer than part $\left.(\nu)\right)$. In particular, since $p_{j}^{\beta}=p_{j}^{\alpha}+(\beta-\alpha) r_{j} \in|X|$ for any $\beta>\alpha$, we see that $r_{j}$ lies in $|\operatorname{rec}(X)|$. It is now easy to see that in fact the line segments in between must also lie in $|\operatorname{rec}(X)|$.

Lemma 3.2. Let $X$ be a d-dimensional polyhedral complex such that $|X|$ is tropically convex. Assume that $\operatorname{rec}(X)=\{\operatorname{rec}(\sigma) ; \sigma \in X\}$ is a fan. Then for each maximal cone $\rho$ of $\operatorname{rec}(X)$, there is exactly one maximal cell $\sigma$ of $X$ such that $\rho=\operatorname{rec}(\sigma)$.

Proof. Assume there are two maximal cells $\sigma, \sigma^{\prime}$ of $X$ such that $\rho=\operatorname{rec}(\sigma)=\operatorname{rec}\left(\sigma^{\prime}\right)$. Pick any two points $p \in \sigma, p^{\prime} \in \sigma^{\prime}$. For $r \in \rho$ we write $q_{r}=p+r, q_{r}^{\prime}=p^{\prime}+r$. As taking the tropical convex hull commutes with translations, we see that

$$
\operatorname{tconv}\left\{q_{r}, q_{r}^{\prime}\right\}=\operatorname{tconv}\left\{p, p^{\prime}\right\}+r .
$$

This implies that $\operatorname{tconv}\left\{p, p^{\prime}\right\}+\rho \subseteq|X|$. But as $p-p^{\prime} \notin V_{\sigma}=V_{\rho}$, there must be a line segment $l \subseteq \operatorname{tconv}\left\{p, p^{\prime}\right\}$, whose slope does not lie in $V_{\sigma}$. Hence $l+\rho$ is a $(d+1)$-dimensional set contained in $|X|$, which is a contradiction to our assumption.

Proposition 3.3. Let $X$ be a tropically convex polyhedral complex and $p \in|X|$. Then $\operatorname{Star}_{X}(p)$ is tropically convex as well. 
Proof. As tropical convexity is preserved under translation, we can assume $p=0$. Let $v, v^{\prime} \in \operatorname{Star}_{X}(0)$. It is clear that for any $\alpha>0, \operatorname{part}\left(\alpha\left(v^{\prime}-v\right)\right)$ does not change. Hence $\operatorname{tconv}\left\{\alpha v, \alpha v^{\prime}\right\}=\alpha \operatorname{tconv}\left\{v, v^{\prime}\right\}$. As $v, v^{\prime} \in \operatorname{Star}_{X}(p)$, the left hand side is contained in $|X|$ for all $\alpha \leqslant 1$. This implies $\operatorname{tconv}\left\{v, v^{\prime}\right\} \subseteq\left|\operatorname{Star}_{X}(p)\right|$.

Definition 3.4. Let $X$ be a polyhedral fan in $\mathbb{R}^{n} / \mathbf{1}$. We write

$$
\mathcal{F}_{X}:=\left\{F \subseteq[n]: v_{F} \in|X|\right\} .
$$

For any chain $\mathcal{C}=\left(F_{1}, \ldots, F_{d}=E\right)$ in $\mathcal{F}_{X}$, we will define cone $(\mathcal{C})$ as in Definition 2.12. Note that each such cone is unimodular and has dimension $d-1$.

We then obtain a polyhedral fan, the chain fan of $X$ :

$$
\mathrm{Ch}_{X}:=\left\{\operatorname{cone}(\mathcal{C}) ; \mathcal{C} \text { a chain in } \mathcal{F}_{X}\right\}
$$

In general this fan obviously need not be pure and can be empty.

Remark 3.5. A special case is $X=\mathbb{R}^{n} / \mathbf{1}$. Then $\mathrm{Ch}_{X}=: \mathrm{Ch}_{n}$ is a subdivision of $\mathbb{R}^{n} / \mathbf{1}$ according to partitions, i.e. two elements lie in the interior of the same cone, if and only if they have the same partition. In particular, if $x \in \mathbb{R}^{n} / \mathbf{1}$, then the minimal cone of $\mathrm{Ch}_{n}$ containing $x$ is $d$-dimensional if and only if het $(x)=d+1$. Note that $\mathrm{Ch}_{n}$ can also be defined as the quotient of the normal fan of the permutohedron or as the chains-of-flats subdivision corresponding to the uniform matroid $U_{n, n}$.

Lemma 3.6. Let $X$ be a tropically convex polyhedral fan in $\mathbb{R}^{n} / \mathbf{1}$. Then the following hold:

1. Assume $\operatorname{dim}(X)=d$ and $x \in|X|$. Then het $(x) \leqslant d+1$. In particular, $X$ is contained in the d-dimensional skeleton of $\mathrm{Ch}_{n}$.

2. $\mathcal{F}_{X}$ is closed under intersections, i.e. if $F, F^{\prime} \in \mathcal{F}_{X}$, then $F \cap F^{\prime} \in \mathcal{F}_{X}$.

3. If $\mathcal{C}$ is any chain in $\mathcal{F}_{X}$, then $\operatorname{cone}(\mathcal{C}) \subseteq|X|$.

Proof. 1. Fix a representative of $x$ and assume that $s:=\operatorname{het}(x)>d+1$. Let $\operatorname{part}(x)=$ $I_{1} \cup \cdots \cup I_{s}$ as in Remark 2.4 and $F_{j}:=\bigcup_{i \leqslant j} I_{i}$. The tropical line segment tconv $\{0, x\}$ consists of segments connecting the points $0=p_{1}, \ldots, p_{s}=x$, where

$$
p_{j}=\sum_{i=2}^{j}\left(x\left(I_{i-1}\right)-x\left(I_{i}\right)\right) e_{F_{i-1}} .
$$

We will show inductively that for $k=2, \ldots, s-1$ there are continuous, concave, piecewise affine linear functions $\epsilon_{k}: \mathbb{R}^{k-1} \rightarrow \mathbb{R}$, such that $\epsilon_{k}$ is strictly positive on $\left(\mathbb{R}_{>0}\right)^{k-1}$ and such that for $k \geqslant 1$ we have

$$
P_{k}=\left\{\sum_{i=1}^{k} \lambda_{i} e_{I_{i}} ; \lambda_{i} \in\left[0, \epsilon_{i}\left(\lambda_{1}, \ldots, \lambda_{i-1}\right)\right] \text { for all } i>1, \lambda_{1} \geqslant 0\right\} \subseteq|X| .
$$


Then $P_{s-1}$ is a polyhedron of dimension $s-1>d$ in the fan $X$, which is a contradiction to our assumption that $\operatorname{dim} X=d$.

For $k=1$ we get $P_{1}=\left\{\lambda_{1} e_{I_{1}} ; \lambda_{1} \geqslant 0\right\}$. As $p_{2}=\left(x\left(I_{1}\right)-x\left(I_{2}\right)\right) e_{I_{1}} \in X$ and $X$ is a fan, this is always contained in $X$. Now assume $k>1$ and that we have found $\epsilon_{2}, \ldots, \epsilon_{k-1}$. Let $q=\sum_{i=1}^{k-1} \lambda_{i} e_{I_{i}} \in P_{k-1}$ and assume $0<\lambda_{i}$ for all $i$. In particular, $q\left(I_{i}\right)>0$ for all $i<k$ and $q\left(I_{i}\right)=0$ for $i \geqslant k$. We now consider the tropical line segment tconv $\left\{\alpha q, p_{k+1}\right\}$, where $\alpha>0$. By induction $q \in|X|$ and since $X$ is a fan, so is $\alpha q$. We conclude that $\operatorname{tconv}\left\{\alpha q, p_{k+1}\right\} \subseteq|X|$. Let $\Delta=p_{k+1}-\alpha q$. Note that $\Delta$ has constant entries on each $I_{j}$ (as both $q$ and $p_{k+1}$ do). More precisely, we have

$$
\Delta\left(I_{j}\right)= \begin{cases}0, & \text { if } j>k \\ x\left(I_{k}\right)-x\left(I_{k+1}\right), & \text { if } j=k \\ x\left(I_{j}\right)-x\left(I_{k+1}\right)-\alpha \lambda_{j}, & \text { if } j<k .\end{cases}
$$

Now, if we pick $\alpha$ sufficiently large, $\Delta$ is maximal on entries in $I_{k}$ : For $j \neq k$ we have

$$
\Delta\left(I_{k}\right)-\Delta\left(I_{j}\right)= \begin{cases}x\left(I_{k}\right)-x\left(I_{k+1}\right)>0, & \text { if } j>k \\ \underbrace{x\left(I_{k}\right)-x\left(I_{j}\right)}_{<0}+\alpha \underbrace{\lambda_{j}}_{>0}, & \text { if } j<k\end{cases}
$$

and the latter is always greater than zero if we pick

$$
\alpha>\max _{j<k}\left\{\frac{x\left(I_{1}\right)-x\left(I_{k+1}\right)}{\lambda_{j}}\right\}=: m_{k, \lambda} .
$$

In this case the first line segment of tconv $\left\{\alpha q, p_{k+1}\right\}$, going from $\alpha q$ to $p_{k+1}$, has slope $e_{I_{k}}$. Its length is $d_{k}:=x\left(I_{k}\right)-x\left(I_{k+1}\right)$ : Our choice of $\alpha$ implies that $\Delta\left(I_{j}\right)<0$ for all $j<k$.

In particular, $\left\{\alpha q+\lambda e_{I_{k}} ; \lambda \in\left[0, d_{k}\right]\right\} \subseteq|X|$. But that implies that $q+\lambda e_{I_{k}} \in|X|$, whenever

$$
0 \leqslant \lambda \leqslant d_{k} \cdot \frac{1}{m_{k, \lambda}}=d_{k} \cdot \min _{j<k}\left\{\lambda_{j} \cdot \frac{1}{x\left(I_{1}\right)-x\left(I_{k+1}\right)}\right\}=: \epsilon_{k}\left(\lambda_{1}, \ldots, \lambda_{k-1}\right) .
$$

As $\epsilon_{k}$ clearly fulfills all desired properties, the claim follows.

2. Follows directly from the fact that $v_{F} \oplus v_{F^{\prime}}=v_{F \cap F^{\prime}}$.

3. Let $\mathcal{C}=\left(F_{1}, \ldots, F_{l}=E\right)$ be a chain in $\mathcal{F}_{X}$. An element of cone $(\mathcal{C})$ is of the form $w=\sum_{i=1}^{l-1} \lambda_{i} v_{F_{i}}$ for some $\lambda_{i} \geqslant 0$. We define $\mu_{j}=\sum_{i=j+1}^{l-1} \lambda_{j}$ for $j=0, \ldots, l-1$ and claim that

$$
w=\bigoplus_{j=1}^{l-1}\left(-\mu_{j}\right) \odot\left(\mu_{0} \cdot v_{F_{j}}\right),
$$


which by assumption is an element of $|X|$. Indeed, let $k \in\{1, \ldots, n\}$ and note that $\mu_{0} \geqslant \mu_{1} \geqslant \ldots \geqslant \mu_{l-1}=0$. Then

$$
\begin{aligned}
\left(\bigoplus_{j=1}^{l-1}\left(-\mu_{j}\right) \odot\left(\mu_{0} \cdot v_{F_{j}}\right)\right)_{k} & =\max _{j=1, \ldots, l-1}\left(\mu_{0} \cdot\left(v_{F_{j}}\right)_{k}-\mu_{j}\right) \\
& = \begin{cases}-\mu_{0}, & \text { if } k \in F_{1} \\
-\mu_{j(k)}, \text { where } j(k):=\max \left\{j: k \notin F_{j}\right\}, & \text { otherwise }\end{cases} \\
& =-\sum_{i: k \in F_{i}} \lambda_{i} \\
& =w_{k} .
\end{aligned}
$$

\section{Tropical convexity and valuated matroids}

By Proposition 2.14 we only need to prove that any tropically convex tropical variety is supported on a tropical linear space. In fact, it will suffice to reduce to the case of fans:

Proposition 4.1. Let $X$ be a tropical variety whose support is a tropically convex fan. Then $|X|=B(M)$ for a matroid $M$.

Our main theorem now follows from this:

Proof. (of Theorem 1.1) The "if" direction follows from Proposition 2.14. For the "only if" direction, let $X$ be a tropical variety with tropically convex support. By Propositions 3.3 and 4.1, $\operatorname{Star}_{X}(p)$ is supported on a matroidal fan $B(M(p))$ at each $p \in|X|$. Since

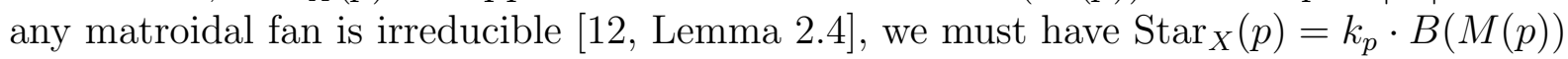
for some $k_{p} \in \mathbb{N}$. As $|X|$ is tropically convex, it is also path-connected. This implies that $k_{p}=k_{p^{\prime}}=: k$ for all $p, p^{\prime} \in|X|$. We conclude that $X$ has constant weight function $\omega_{X} \equiv k$ for some $k \in \mathbb{N}$, so we can write it as $X=k \cdot Y$, where $|X|=|Y|$ and $Y$ has constant weight 1.

By Propositions 3.1 and $4.1, \operatorname{rec}(X)$ is also supported on a matroidal fan and hence of the form $l \cdot B(M)$ for some $l \in \mathbb{N}$ and some matroid $M$. Using Lemma 3.2, we see that $\operatorname{rec}(Y)=B(M)$ (and in fact: $k=l$ ). By Theorem 2.11 we must have $Y=B(M, w)$ for some valuation $w$ on $M$, so $|X|=|Y|=|B(M, w)|$, as claimed.

The general idea for proving Proposition 4.1 is to revert the procedure described in Remark 2.13: We define $M$ via its flats, which is the set $\mathcal{F}_{X}$ of all sets whose incidence vectors lie in $|X|$. We show that $X$ is supported on the fan of chains of $\mathcal{F}_{X}$. Then it only remains to show that $\mathcal{F}_{X}$ actually fulfills the axioms required for a set of flats. Naturally, the balancing condition plays a crucial role in the proofs of both statements.

Proposition 4.2. Let $X$ be a tropical fan and assume $|X|$ is tropically convex. Then

$$
|X|=\left|\mathrm{Ch}_{X}\right| \text {. }
$$


Proof. Let $d:=\operatorname{dim} X$ and let $\mathcal{X}$ be a polyhedral structure of $X$. By Lemma 3.6,(1), $|X|$ is contained in the $d$-dimensional skeleton of $\mathrm{Ch}_{n}$. By intersecting $X$ with $\mathrm{Ch}_{n}$, we can now assume that each cone of $\mathcal{X}$ is contained in some $d$-dimensional cone $(\mathcal{C})$, where $\mathcal{C}$ is a chain of arbitrary subsets of $[n]$.

The balancing condition of $X$ now dictates that if $\rho=\operatorname{cone}(\mathcal{C}) \in \mathrm{Ch}_{n}$ contains a maximal cone of $\mathcal{X}$ in its interior, all of $\rho$ must be in $|X|$ : Otherwise, it would contain a codimension one face $\tau$ of $\mathcal{X}$ in its interior, such that there is one maximal cone $\sigma>\tau$ with $\sigma \subseteq \rho$ but no other maximal cone $\sigma^{\prime}>\tau$ is contained in $\rho$. Balancing implies that at least one other maximal cone $\sigma^{\prime}$ is adjacent to $\tau$. By our previous argument, $\sigma$ and $\sigma^{\prime}$ both lie in $d$-dimensional cones of $\mathrm{Ch}_{n}$. But these cones intersect only in their boundary.

This proves $|X| \subseteq\left|\mathrm{Ch}_{X}\right|$ and the converse follows from Lemma 3.6,(3).

Proof. (of Proposition 4.1)

Proposition 4.2 tells us that we can equip $X$ with the polyhedral structure of $\mathrm{Ch}_{X}$ : Balancing implies that all cones contained in a cone of $\mathrm{Ch}_{X}$ must have the same weight. Hence it is sufficient to show that $\mathcal{F}_{X}$ defines indeed a set of flats of a matroid. More precisely, we have to show the following:

1. $E \in \mathcal{F}_{X}$.

2. If $F, F^{\prime} \in \mathcal{F}_{X}$, then $F \cap F^{\prime} \in \mathcal{F}_{X}$.

3. Let $F \in \mathcal{F}_{X}$ and assume $F_{1}, \ldots, F_{k}$ are the minimal elements of $\mathcal{F}_{X} \backslash\{F\}$ that contain $F$. Then $E \backslash F$ is the disjoint union of $F_{1} \backslash F, \ldots, F_{k} \backslash F$.

The first statement is trivial and the second follows from Lemma 3.6, (2).

To prove the third axiom, let $F \in \mathcal{F}_{X}$ and denote by $F_{1}, \ldots, F_{k}$ the minimal elements of $\mathcal{F}_{X}$ containing it. Then $\left(F_{i} \backslash F\right) \cap\left(F_{j} \backslash F\right)=\emptyset$ by minimality and the second axiom. Hence we only have to prove that $\bigcup_{i=1}^{k} F_{i}=E$. By Proposition 4.2 and the fact that $X$ is pure of some dimension $d$, every maximal chain in $\mathcal{F}_{X}$ has the same length $d+1$. Let $G \in \mathcal{F}_{X}$ and $\mathcal{C}=\left(F_{1}, \ldots, F_{d+1}=E\right)$ a maximal chain in $\mathcal{F}_{X}$. We define the rank of $G$ to be $\operatorname{rank}(G):=i$, if $F_{i}=G$. This is independent of the actual chain: Otherwise we could combine two chains with $G$ occurring at different positions to form a chain of length greater than $d+1$.

We will now prove the last axiom by induction on $c(F):=d+1-\operatorname{rank}(F)$. If $c(F)=1$, then $k=1$ and $F_{1}=E$, so the statement is true. Now let $c(F)>1$ and $j \in E \backslash F$. By induction, there exists an $F^{\prime} \in \mathcal{F}_{X}$ of $\operatorname{rank} \operatorname{rank}(F)+2$ and with $F \subseteq F^{\prime}$, such that $j \in F^{\prime}$. We can now pick a chain

$$
\mathcal{D}=\left(G_{1}, \ldots, G_{c(F)}=F, G_{c(F)+2}=F^{\prime}, \ldots, G_{d+1}=E\right),
$$

where $\operatorname{rank}\left(G_{i}\right)=i$ for all $i$ (such a chain exists, as $X$ is pure). Then $\operatorname{cone}(\mathcal{D})$ is a codimension one cone. Using the fact that all chain cones are unimodular, the balancing equation at $\operatorname{cone}(\mathcal{D})$ reads:

$$
v:=\sum_{G \in \mathcal{G}(\mathcal{D})} w_{G} v_{G} \in V_{\text {cone }(\mathcal{D})}
$$


where $\mathcal{G}(\mathcal{D}):=\left\{G \in \mathcal{F}_{X} ; F \subsetneq G \subsetneq F^{\prime}\right\} \subseteq\left\{F_{1}, \ldots, F_{k}\right\}$ and $w_{G} \in \mathbb{Z} \backslash\{0\}$ denotes the weight of the corresponding maximal cone.

As $v \in V_{\text {cone }(\mathcal{D})}$, all entries $\left\{v_{i}, i \in F^{\prime} \backslash F\right\}$ agree (this notion is obviously well-defined in $\mathbb{R}^{n} / \mathbf{1}$ ). If we pick the obvious representative $-\sum_{i \in G} e_{i} \in \mathbb{R}^{n}$ for each $v_{G}$ and use the fact that for any $F_{s}, F_{t} \in \mathcal{G}(\mathcal{D})$ we have $\left(F_{s} \backslash F\right) \cap\left(F_{t} \backslash F\right)=\emptyset$, then for $i \in F^{\prime} \backslash F$ we have

$$
v_{i}= \begin{cases}-w_{G}, & \text { if there is a } G \in \mathcal{G}(D) \text { with } i \in G \\ 0, & \text { otherwise. }\end{cases}
$$

As $X$ is pure, $\mathcal{G}(\mathcal{D})$ is not empty. But this implies that there must be an $F_{s} \in \mathcal{G}(\mathcal{D})$ with $j \in F_{s}$.

\section{Local-to-global tropical convexity}

In classical convexity theory, there are various local-to-global principles. In this section, we prove Theorem 1.2, a tropical analogue of a result proven by Tietze and Nakajima $[26,20]$. It states that any closed connected subset of $\mathbb{R}^{n}$, which is locally convex, is already convex. The main strategy of the proof follows a standard argument for classical convexity - though there is some extra work involved due to the fact that $\mathbb{R}^{n} / \mathbf{1}$ is not uniquely geodesic with its canonical metric (see Remark 5.3).

Definition 5.1. Let $x \in \mathbb{R}^{n} / \mathbf{1}$. We define the tropical norm of $x$ to be

$$
\|x\|_{\text {trop }}:=\max \left\{x_{i}\right\}-\min \left\{x_{i}\right\} .
$$

We also fix the following notations:

$$
\begin{aligned}
B_{r}^{\operatorname{trop}}(x) & :=\left\{y \in \mathbb{R}^{n} / \mathbf{1} ;\|y-x\|_{\text {trop }} \leqslant r\right\}, \\
\partial B_{r}^{\operatorname{trop}}(x) & :=\left\{y \in \mathbb{R}^{n} / \mathbf{1} ;\|y-x\|_{\text {trop }}=r\right\}, \\
I_{\min }(x) & :=\left\{i \in[n] ; x_{i} \text { minimal }\right\}, \\
I_{\max }(x) & :=\left\{i \in[n] ; x_{i} \text { maximal }\right\} .
\end{aligned}
$$

For a compact set $S$ and a point $x$, we will also write

$$
\|x-S\|_{\text {trop }}:=\min \left\{\|x-s\|_{\text {trop }} ; s \in S\right\} .
$$

The following all have easy and elementary proofs:

Lemma 5.2. 1 . $\|\cdot\|_{\text {trop }}$ is twice the quotient norm of the maximum norm on $\mathbb{R}^{n}$. In particular, it defines a norm on the $\mathbb{R}$-vector space $\mathbb{R}^{n} / \mathbf{1}$.

2. Let $r>0$ and $P_{r, n}$ be the cube in $\mathbb{R}^{n}$ with vertices $r e_{F}, \emptyset \subseteq F \subseteq[n]$. Then $B_{r}^{\text {trop }}(0)$ in $\mathbb{R}^{n} / \mathbf{1}$ is the image of $P_{r, n}$ under the quotient map.

3. $B_{r}^{\mathrm{trop}}(x)$ is a polytrope, i.e. convex and tropically convex. 
4. If $x \in \mathbb{R}^{n} / \mathbf{1}$ and $\operatorname{tconv}\{0, x\}$ consists of actual line segments connecting points $0=p_{1}, \ldots, p_{s}=x$, then

$$
\|x\|_{\text {trop }}=\sum_{i=1}^{s-1}\left\|p_{i+1}-p_{i}\right\|_{\text {trop }}
$$

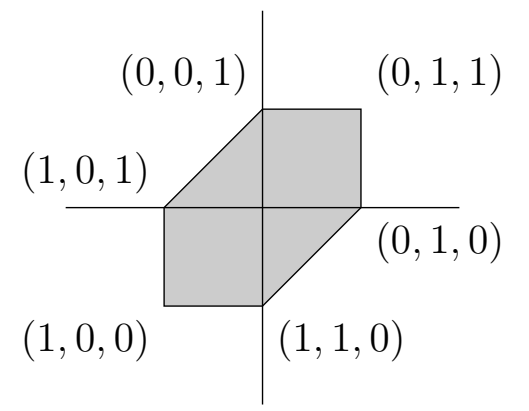

Figure 3: The unit sphere $B_{1}^{\text {trop }}(0)$ in $\mathbb{R}^{3} / \mathbf{1}$. The picture is drawn in two dimensions by setting the first coordinate to 0 .

Remark 5.3. The tropical norm was already introduced in [6] to study tree metrics. Joswig shows that $\mathbb{R}^{n} / \mathbf{1}$ is a geodesic space [15]: The tropical line segment between two points is a geodesic with respect to the metric induced by $\|\cdot\|_{\text {trop }}$. However, it is not uniquely geodesic. There are generally various paths from $x$ to $y$ whose length is $\|x-y\|_{\text {trop }}$ (see also Figure 4).

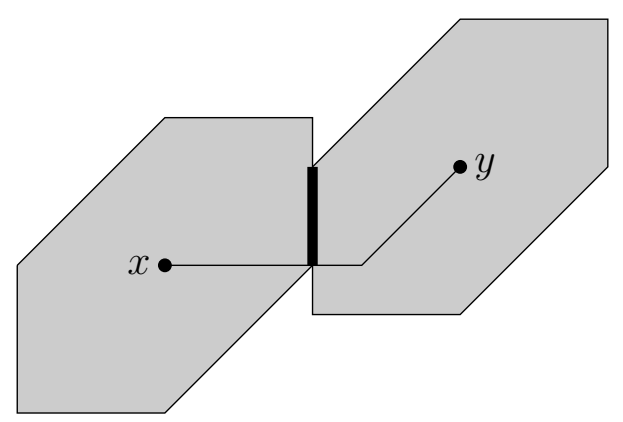

Figure 4: The set of points $z$ with $\|x-z\|_{\text {trop }}=\|z-y\|_{\text {trop }}=\|x-y\|_{\text {trop }} / 2$ is a polytrope.

Lemma 5.4. Let $x, y, z \in \mathbb{R}^{n} /$ 1. Then for any point $p \in \operatorname{tconv}\{x, y\}$, we have

$$
I_{\max }(p-z) \subseteq I_{\max }(x-z) \cup I_{\max }(y-z) .
$$

Proof. Let $j \in I_{\max }(p-z)$ and assume $j \notin I_{\max }(x-z)$. We know that we can write $y=x+\sum_{i=1}^{s} \alpha_{i} e_{F_{i}}$, where $\alpha_{i}>0$ and $F_{i} \subsetneq F_{i+1}$ for all $i$ and each summand corresponds to a vertex on the tropical line segment $\operatorname{tconv}\{x, y\}$. Hence $p=x+\sum_{i=1}^{k-1} \alpha_{i} e_{F_{i}}+\beta e_{F_{k}}$ for 
some $k \leqslant s$ and $\beta \leqslant \alpha_{k}$. Since $p_{j}-z_{j}=\left(x_{j}-z_{j}\right)+\sum_{i=1}^{k-1} \alpha_{i}\left(e_{F_{i}}\right)_{j}+\beta\left(e_{F_{k}}\right)_{j}$ is maximal and $x_{j}-z_{j}$ is not maximal, we must have that $j \in F_{m}$ for some $m \leqslant k$. In particular, $j \in F_{l}$ for all $l \geqslant k$, so $y_{j}-z_{j}$ is still maximal.

Definition 5.5. Let $X \subseteq \mathbb{R}^{n} / 1$ and $x, y \in X$.

- We call $X$ locally tropically convex, if for every $x \in X$ there exists an $\epsilon>0$, such that $B_{\epsilon}^{\operatorname{trop}}(x) \cap X$ is tropically convex.

- A tropical path in $X$ from $x$ to $y$ is an injective continuous map $\gamma:[0,1] \rightarrow X$, whose image is a concatenation of tropical line segments leading from $x$ to $y$. The length $l(\gamma)$ of $\gamma$ is the length with respect to $\|\cdot\|_{\text {trop }}$, i.e. if $\gamma$ consists of tropical line segments connecting $x=x_{0}, \ldots, x_{k}=y$, then

$$
l(\gamma)=\sum_{i=1}^{k}\left\|x_{i}-x_{i-1}\right\|_{\text {trop }}
$$

- We define the distance of $x$ and $y$ in $X$ to be

$$
d_{X}(x, y):=\inf \{l(\gamma) ; \gamma \text { a tropical path from } x \text { to } y .\}
$$

Lemma 5.6. Let $X \subseteq \mathbb{R}^{n} / \mathbf{1}$ be locally tropically convex and $x, y \in X$. Assume there is a point $z \in X$ such that the following hold:

- $d_{X}(x, z)=d_{X}(z, y)=d_{X}(x, y) / 2$.

- $\|z-\operatorname{tconv}\{x, y\}\|_{\text {trop }}$ is minimal among all points fulfilling the first property.

- $\operatorname{tconv}\{x, z\}, \operatorname{tconv}\{y, z\} \subseteq X$.

Then $z \in \operatorname{tconv}\{x, y\}$, so $\operatorname{tconv}\{x, y\} \subseteq X$.

Proof. Assume $z \notin \operatorname{tconv}\{x, y\}$. We define $F:=I_{\max }(x-z), F^{\prime}:=I_{\max }(y-z)$. Then $e_{F}, e_{F^{\prime}}$ are the outgoing slopes of the tropical line segments from $z$ to $x$ and $y$, respectively (see also Figure 5 for an illustration).

Now choose $\epsilon>0$ small and let

$$
z^{\prime}:=\left(z+\epsilon e_{F}\right) \oplus\left(z+\epsilon e_{F^{\prime}}\right)=z+\epsilon e_{F \cup F^{\prime}} .
$$

By local tropical convexity, this lies in $X$ for sufficiently small $\epsilon$.

First of all, we see that $z^{\prime}$ still fulfills the first property: Note that the concatenation of $\operatorname{tconv}\left\{x, z+\epsilon e_{F}\right\}$ and $\operatorname{tconv}\left\{z+\epsilon e_{F}, z^{\prime}\right\}$ forms a tropical path in $X$ from $x$ to $z^{\prime}$. Again assuming $\epsilon$ to be sufficiently small and using that $x \neq z$, we get

$$
\begin{aligned}
d_{X}\left(x, z^{\prime}\right) & \leqslant\left\|x-\left(z+\epsilon e_{F}\right)\right\|_{\text {trop }}+\left\|z^{\prime}-\left(z+\epsilon e_{F}\right)\right\|_{\text {trop }} \\
& \leqslant\left(\|x-z\|_{\text {trop }}-\epsilon\right)+\epsilon \\
& =\|x-z\|_{\text {trop }}=d_{X}(x, z) .
\end{aligned}
$$


Similarly, $d_{X}\left(y, z^{\prime}\right) \leqslant d_{X}(y, z)$. But as $z$ was already a midpoint, this implies equality.

We now claim that $\left\|z^{\prime}-\operatorname{tconv}\{x, y\}\right\|_{\text {trop }}<\|z-\operatorname{tconv}\{x, y\}\|_{\text {trop }}=: l$, which is a contradiction to our assumption. To see this, let

$$
M:=\left\{p \in \operatorname{tconv}\{x, y\} ;\|z-p\|_{\text {trop }}=l\right\} .
$$

As $M=\operatorname{tconv}\{x, y\} \cap B_{l}^{\operatorname{trop}}(z)$, it is tropically convex. We know by Lemma 5.4 , that for any point $p$ in $M$, we have $I_{\min }(z-p) \subseteq F \cup F^{\prime}$. Also note that by assumption $z \notin M$. We will now prove that $I_{\max }(z-p) \cap\left(F \cup F^{\prime}\right)=\emptyset$.

Assume $M=\{p\}$ is only a point. Then moving from $p$ along the tropical line segment tconv $\{x, y\}$ strictly increases the distance to $z$. Let $G:=I_{\max }(x-p), G^{\prime}:=I_{\max }(y-p)$. Then for small $\epsilon^{\prime}$ we must have

$$
\left\|z-\left(p+\epsilon^{\prime} e_{G}\right)\right\|_{\text {trop }}=\left\|(z-p)-\epsilon^{\prime} e_{G}\right\|_{\text {trop }}>\|z-p\|_{\text {trop }} .
$$

So if $\emptyset \neq I_{\max }(z-p) \cap F=I_{\max }(z-p) \cap I_{\max }(x-z)$, then we must have $G=I_{\max }(z-p) \cap F$. But then

$$
\left\|z-\left(p+\epsilon^{\prime} e_{G}\right)\right\|_{\text {trop }} \leqslant\|z-p\|_{\text {trop }}
$$

which is a contradiction. The same argument works for $F^{\prime}$ and $G^{\prime}$, so we see that $I_{\max }(z-$ $p) \cap\left(F \cup F^{\prime}\right)=\emptyset$.

If $M$ is a tropical line segment, we can choose $p$ such that tconv $\{x, y\}$ is locally at $p$ a line with slope $v_{G}, G:=I_{\max }(x-p)$ and

$$
\left\|z-\left(p \pm \epsilon^{\prime} e_{G}\right)\right\|_{\text {trop }}=\|z-p\|_{\text {trop }}
$$

But this is only possible if either $I_{\min }(z-p) \cup I_{\max }(z-p) \subseteq G$ or $\left(I_{\min }(z-p) \cup I_{\max }(z-\right.$ $p)) \cap G=\emptyset$. So if $\emptyset \neq I_{\max }(z-p) \cap F$, we must again have $G=I_{\max }(z-p) \cap F$, so neither of the above two possibilities would hold. Again, the same argument works for $F^{\prime}$.

In either case, we see that $I_{\max }(z-p) \cap\left(F \cup F^{\prime}\right)=\emptyset$ and $I_{\min }(z-p) \subseteq\left(F \cup F^{\prime}\right)$. But then

$$
\left\|z^{\prime}-p\right\|_{\text {trop }}=\left\|(z-p)+\epsilon e_{F \cup F^{\prime}}\right\|_{\text {trop }}<\|z-p\|_{\text {trop }} .
$$

This contradicts our assumption. Hence we must have $z \in \operatorname{tconv}\{x, y\}$.

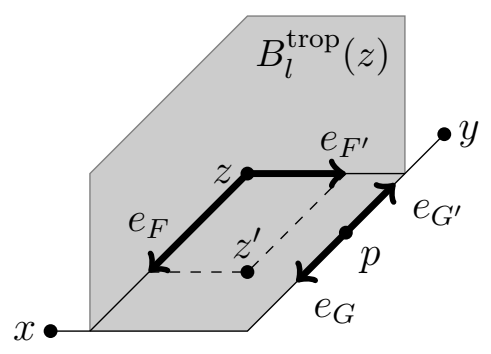

Figure 5: Constructing a point closer to the tropical line segment using local tropical convexity. 
Proof. (of Theorem 1.2) Let $x, y \in X$ and set $r:=d_{X}(x, y)$.

Since $X$ is closed and locally tropically convex, there exists a midpoint, i.e. a point $z \in X$ such that

$$
d_{X}(x, z)=d_{X}(y, z)=\frac{1}{2} r .
$$

Note that the set of midpoints of $x$ and $y$ is a compact set. It is obviously closed and must be a subset of $B_{r / 2}^{\text {trop }}(x) \cup B_{r / 2}^{\text {trop }}(y)$. Hence we can choose $z$ to have minimal distance to $\operatorname{tconv}\{x, y\}$.

In this manner, we recursively construct points $z_{i, n} \in X$ with $1 \leqslant n, 0 \leqslant i \leqslant 2^{n}$, such that

- $z_{0, n}=x, z_{n, n}=y$ and $z_{i, n}=z_{2 i, n+1}$.

- $z_{2 i+1, n+1}$ is a midpoint of $z_{i, n}$ and $z_{i+1, n}$ and it has minimal distance to the tropical line segment $\operatorname{tconv}\left\{z_{i, n}, z_{i+1, n}\right\}$.

In particular, we have $d_{X}\left(z_{i, n}, z_{i+1, n}\right)=r / 2^{n}$. Now we have

$$
\left\|z_{i, n}-x\right\|_{\text {trop }} \leqslant d_{X}\left(z_{i, n}, x\right) \leqslant\left(i / 2^{m}\right) r \leqslant r
$$

for all $i$ and $n$, so $z_{i, n} \in B_{r}^{\text {trop }}(x) \cap X=: B$, which is a compact set. Hence we can choose a global $\delta>0$ such that for all $x \in B$, the set $B_{\delta}^{\text {trop }}(x) \cap X$ is tropically convex.

By choosing $n$ large enough, we can now assume that $r / 2^{n+1}<\delta$. Then for each $i$, $B_{\delta}^{\text {trop }}\left(z_{2 i+1, n+1}\right)$ contains both $z_{i, n}$ and $z_{i+1, n}$, so their tropical convex hull is contained in $X$. Applying Lemma 5.6 inductively, we see that $\operatorname{tconv}\{x, y\} \subseteq X$.

Corollary 5.7. Let $X$ be a connected tropical variety in $\mathbb{R}^{n} / \mathbf{1}$, which is locally a multiple of a matroidal fan, i.e. $\operatorname{Star}_{X}(p)=k_{p} \cdot B(M(p))$ for each $p \in X$, some $k_{p} \in \mathbb{Z}$ and some matroid $M(p)$. Then $X$ is supported on a tropical linear space.

\section{Acknowledgements}

I would like to thank Michael Joswig for many helpful suggestions.

\section{References}

[1] Xavier Allamigeon, Pascal Benchimol, Stéphane Gaubert, and Michael Joswig. Tropicalizing the simplex algorithm. SIAM J. Opt. 24.4:2096-2117, 2014.

[2] Xavier Allamigeon, Stéphane Gaubert, and Michael Joswig. Combinatorial simplex algorithms can solve mean payoff games. SIAM J. Discrete Math. 29.2:751-795, 2015. arXiv: 1309.5925

[3] Lars Allermann, Simon Hampe, and Johannes Rau. On rational equivalence in tropical geometry. Preprint, 2014. to appear in: Canadian Journal of Mathematics arXiv: 1408.1537 
[4] Frederico Ardila and Carly Klivans. The bergman complex of a matroid and phylogenetic trees. J. Comb. Theory, Ser. B, 96:38-49, 2006. arXiv:math/0311370v2

[5] Florian Block and Josephine Yu. Tropical convexity via cellular resolutions. Journal of Algebraic Combinatorics, 24(1):103-114, 2006. arXiv:math/0503279

6] Mike Develin and Bernd Sturmfels. Tropical convexity. Doc. Math., 9:1-27, 2004. arXiv: math/0308254

[7] Mike Develin and Josephine Yu. Tropical polytopes and cellular resolutions. Experimental Mathematics, 16(3):277-292, 2007. arXiv:math/0605494

[8] Andreas W.M Dress and Walter Wenzel. Valuated matroids. Advances in Mathematics, 93(2):214 - 250, 1992.

[9] Eva Maria Feichtner and Bernd Sturmfels. Matroid polytopes, nested sets and bergman fans. Portugaliae Mathematica. Nova Srie, 62(4):437-468, 2005.

[10] Alex Fink. Tropical cycles and chow polytopes. Beitrge zur Algebra und Geometrie - Contributions to Algebra and Geometry, 54(1):13-40, 2013. arXiv:1001.4784

[11] Alex Fink and Felipe Rincón. Stiefel tropical linear spaces. J. Combin. Theory Ser. A 135:291-331,2015. arXiv:1305.6329

[12] Georges François and Johannes Rau. The diagonal of tropical matroid varieties and cycle intersections. Collect. Math., 64(2):185-210, 2013. arXiv:1012.3260

[13] Stéphane Gaubert and Ricardo D. Katz. Minimal half-spaces and external representation of tropical polyhedra. J. Algebraic Combin., 33(3):325-348, 2011. arXiv:0908.1586

[14] Ilia Itenberg, Grigory Mikhalkin, and Eugenii Shustin. Tropical Algebraic Geometry. Oberwolfach Seminars Series. Springer Verlag NY, 2007.

[15] Michael Joswig. Essentials of Tropical Combinatorics. Work in progress. http: //page.math.tu-berlin.de/ joswig/etc/index.html

[16] Michael Joswig and Georg Loho. Weighted digraphs and tropical cones. Preprint, 2015. arXiv:1503.04707

[17] Diane Maclagan and Bernd Sturmfels. Introduction to Tropical Geometry, volume 161 of Graduate Studies in Mathematics. American Mathematical Society, Providence, RI, 2015.

[18] Grigory Mikhalkin and Johannes Rau. Tropical Geometry. Work in progress. https: //www.dropbox.com/s/9lpv86oz5f4za75/main.pdf

[19] Kazuo Murota and Akihisa Tamura. On circuit valuation of matroids. Advances in Applied Mathematics, 26(3):192 - 225, 2001.

[20] Souji Nakajima. ber konvexe Kurven und Flchen. Tohoku Mathematical Journal, 29:227-230, 1928.

[21] James Oxley. Matroid Theory. Oxford University Press, New York, 2011.

[22] Johannes Rau. Tropical intersection theory and gravitational descendants. PhD thesis, 2009. http://kluedo.ub.uni-kl.de/volltexte/2009/2370/ 
[23] Felipe Rincón. Isotropical linear spaces and valuated Delta-matroids. J. Combin. Theory Ser. A, 119(1):14-32, 2012. arXiv:1004.4950

[24] David E. Speyer. Tropical linear spaces. SIAM J. Discrete Math., 22(4):1527-1558, 2008. arXiv:math/0410455

[25] Bernd Sturmfels. Solving Systems of polynomial equations. CBMS Regional Conference Series in Mathematics 97, American Mathematical Society, Providence, 2002.

[26] Heinrich Tietze. Über Konvexheit im kleinen und im großen und über gewisse den Punkten einer Menge zugeordnete Dimensionszahlen. Math. Z., 28(1):697-707, 1928. 